\title{
Immuntherapie des malignen Melanoms
}

\author{
Immunotherapy for Melanoma
}

Autor

Institut

\section{E. S. Schultz}

Hautklinik am Klinikum Nürnberg-Nord

\section{Bibliografie}

DOI http://dx.doi.org/

10.1055/s-0029-1244085

Online-Publikation: 1.4. 2010

Akt Dermatol 2010; 36:

250-254 @ Georg Thieme

Verlag KG Stuttgart · New York ISSN 0340-2541

Korrespondenzadresse Prof. Dr. med. Erwin S. Schultz Hautklinik am Klinikum Nürnberg-Nord Prof.-Ernst-Nathan-Str. 1 90419 Nürnberg Erwin.Schultz@klinikumnuernberg.de

\section{Zusammenfassung $\nabla$}

Das Melanom ist ein immunogener Tumor, welcher von den Immunzellen erkannt und zielgenau bekämpft werden kann. Dabei spielen neben den tumorreaktiven T-Lymphozyten auch B-Lymphozyten und NK-Zellen eine wichtige Rolle. Dank der fortschreitenden Erforschung der molekularen Mechanismen der Interaktion von Tumorzellen und Immunsystem werden die immunthera-

\section{Einleitung \\ $\nabla$}

Die Inzidenz des malignen Melanoms hat sich in den letzten drei Jahrzehnten in Deutschland mehr als verdreifacht. Jährlich erkranken etwa 15000 Patienten, wobei das Melanom bei den Frauen mittlerweile den vierthäufigsten bösartigen Tumor überhaupt ausmacht und bei den Männern an sechster Stelle rangiert. Während das Melanom in den meisten Fällen durch eine Operation geheilt werden kann, verschlechtert sich die Prognose mit zunehmender Tumorausbreitung. So beträgt das mittlere Überleben der Patienten mit Fernmetastasen je nach Lokalisation und Ausmaß der Metastasierung zwischen 6 und 12 Monaten. Es besteht folglich ein dringender Bedarf an neuen Therapieansätzen für Patienten mit fortgeschrittenem Tumorleiden. Dies spiegelt sich auch in der zunehmenden Anzahl klinischer Studien mit einer Vielzahl von neuen Wirkstoffen wider. Im Gegensatz zu anderen soliden Tumoren konnte aber bislang keines der neuen Therapeutika die Zulassung für die Behandlung des fortgeschrittenen Melanoms erhalten. Ein seit langem schon verfolgter und immer noch aktueller Ansatz stellt die Immuntherapie dar. Es ist unstrittig, dass das Melanom ein immunogener Tumor ist, welcher von den Zellen des Immunsys- peutischen Interventionen laufend verfeinert. Ein nachhaltiger therapeutischer Erfolg steht bislang noch aus, was nicht zuletzt den zahlreichen „immune escape“-Mechanismen des Melanoms geschuldet ist. In Zukunft wird es im Sinne einer individualisierten Medizin darauf ankommen, Patienten zum Beispiel anhand ihres HLA-Musters und/oder des Antigenexpressionsprofils ihres Tumors zu selektieren, um so das Potenzial der Immuntherapie voll auszuschöpfen.

tems erkannt und erfolgreich bekämpft werden kann. Schon vor der Entdeckung von Tumorantigenen beim Melanom gelang es durch die Gabe von hochdosiertem Interleukin-2 erstaunliche Therapieerfolge mit zum Teil lang anhaltenden Remissionen zu erzielen, was schließlich auch zur Zulassung dieser Substanz in den USA geführt hat. Anfang der 90er-Jahre begann dann die Identifizierung der antigenen Zielstrukturen der antitumoralen Immunantwort als Basis für eine zielgerichtete Immunintervention im Kampf gegen das Melanom. In den letzten 10 Jahren konnten zahlreiche Studien durch unterschiedliche immuntherapeutische Interventionen die erfolgreiche Induktion von Tumorantigen-spezifischen T-Zellen im Blut der Patienten demonstrieren, welche in vielen Fällen eindeutig mit dem klinischen Ansprechen korrelierte. Obwohl bislang der entscheidende therapeutische Durchbruch ausgeblieben ist, sind die oben beschriebenen Beobachtungen doch Grund genug, weiter an der Entwicklung neuer immuntherapeutischer Konzepte zu arbeiten.

Dieser Artikel soll einen Überblick über die verschiedenen Ansätze unter Würdigung der bislang publizierten Daten zur klinischen Wirksamkeit ermöglichen. 


\section{Interferon-alpha}

7

In der adjuvanten Therapiesituation ist Interferon-alpha die einzige in Deutschland zugelassene Substanz und gilt als Standardtherapie, welche in der aktuell gültigen deutschen Leitlinie empfohlen wird. Die optimale Dosis und die Behandlungsdauer bleiben trotz zahlreicher Studien mit vielen tausenden Patienten weiterhin umstritten. Während in den USA bevorzugt die Hochdosistherapie über $1 \mathrm{Jahr}$ durchgeführt wird, kommt in Europa überwiegend die Mittelhochdosis- bzw. Niedrigdosistherapie zum Einsatz. Nach mehr als 20 Jahren klinischer Forschung mit Interferon-alpha gibt es immer noch keinen weltweit einheitlichen Standard.

Immerhin herrscht allgemeiner Konsens über die Tatsache, dass die Gabe von Interferon-alpha das rezidivfreie Überleben für Patienten mit Hochrisikomelanomen signifikant verlängert. Dies wurde für die Niedrigdosistherapie mit $3 \times 3$ Mio I.E. s.c. pro Woche über 18 Monate bei Patienten mit einer Primärtumordicke von $>1,5 \mathrm{~mm}$ sowohl in einer französischen als auch in einer österreichischen Studie gezeigt [1,2]. Im Stadium III nach Resektion von Lymphknotenmetastasen wurde in der EORTC-Studie 18991 mit insgesamt 1256 Patienten ebenfalls eine signifikante Verlängerung des rezidivfreien Überlebens für den Behandlungsarm mit pegyliertem Interferon-alpha-2b beobachtet [3]. Subgruppenanalysen ergaben, dass insbesondere Patienten mit einer Mikrometastase im Lymphknoten (N1) von der Therapie profitierten, nicht aber Patienten mit Makrometastasen bzw. mehreren befallenen Lymphknoten. Eine signifikante Verlängerung des Gesamtüberlebens konnte allerdings keine der oben genannten Studien demonstrieren.

Dies gelang hingegen in der US-amerikanischen E1684-Studie, in der insgesamt 287 Patienten im Stadium IIB und III zwischen Beobachtungsarm und Hochdosistherapie (20 Mio I.E./m2/d i.v. für 5 Tage die Woche über insgesamt 4 Wochen, im Anschluss daran $3 \times$ pro Woche $10 \mathrm{Mio}$ I.E./m2/d s.c. für 48 Wochen) randomisiert wurden. In dieser Studie konnte nicht nur ein signifikant längeres rezidivfreies Überleben, sondern auch eine Verlängerung des Gesamtüberlebens durch die Hochdosistherapie gezeigt werden [4]. Dieser Vorteil war allerdings in einer gepoolten Analyse der Patientendaten aus vier verschiedenen Hochdosisstudien nicht mehr signifikant [5].

In einer 2003 publizierten Metaanalyse von 12 Studien zur adjuvanten Interferontherapie inklusive Hochdosis- und Niedrigdosistherapie konnten Wheatley u. Mitarb. erneut bestätigen, dass die Gabe von Interferon-alpha das rezidivfreie Überleben signifikant verlängert, wobei das Risiko des Rezidives durchschnittlich um $7 \%$ reduziert wird [6].

In einer weiteren Metaanalyse derselben Arbeitsgruppe, in der individuelle Patienten über einen längeren Zeitraum nachverfolgt wurden, zeigte sich ebenfalls eine signifikante Verbesserung des rezidivfreien Überlebens, darüber hinaus aber auch ein statistisch signifikanter Vorteil hinsichtlich des Gesamtüberlebens, welcher mindestens 3\% nach 5 Jahren betrug. Dieser Effekt war dabei weder von der verwendeten Dosis noch von der Therapiedauer abhängig [7].

\section{Was lässt sich daraus für die Praxis schlussfolgern? \\ $\nabla$}

Die adjuvante, niedrigdosierte Immuntherapie mit $3 \times 3$ Mio. I.E. Interferon-alpha über 18 Monate wird bislang in Deutschland ab einer Tumordicke von $\geq 1,5 \mathrm{~mm}$ durchgeführt, wobei internatio- nal zunehmend erst ab einer Tumordicke von $>2 \mathrm{~mm}$ behandelt wird, da die Prognose der Patienten mit einer Tumordicke von 1,01 - 2 mm ohne Ulzeration des Primärtumores mit einer 5-Jahres-Überlebensrate von $89 \%$ sehr gut ist. Besonders deutlich profitieren Patienten mit ulzeriertem Primärtumor von der Interferontherapie, welche auch bei Vorliegen einer Tumordicke von $<2 \mathrm{~mm}$ behandelt werden sollten.

Im Falle der lokoregionären Lymphknotenmetastasierung wird in der Regel ebenfalls eine adjuvante Immuntherapie mit Interferon-alpha angeboten, wobei unterschiedliche Dosierschemata zum Einsatz kommen. Dabei wird in Europa häufig die Mittelhochdosis- und Niedrigdosistherapie bevorzugt, während in den USA die Hochdosistherapie über $1 \mathrm{Jahr}$ als Standard etabliert ist. Es hat sich gezeigt, dass insbesondere Patienten mit einer Mikrometastasierung in den regionären Lymphknoten von der Interferontherapie profitieren, während der Nutzen bei fortgeschrittener Metastasierung weniger gut belegt ist. Schließlich gibt es derzeit keine Evidenz dafür, dass eine längere Therapiedauer über mehrere Jahre die Wirksamkeit der adjuvanten Therapie verbessert.

Beim Vorliegen von Fernmetastasen spielt Interferon-alpha bisher keine therapeutische Rolle.

\section{Anti-CTLA4-Antikörper}

$\nabla$

Um den Organismus vor einer dauerhaften und unkontrollierten Aktivierung von T-Lymphozyten und damit Autoimmunität zu schützen, hat sich die Natur eine Art immunologischer Bremse ausgedacht. T-Lymphozyten exprimieren nach Aktivierung vermehrt ein Typ-I-Transmembranprotein namens CTLA-4 (CD152), welches zur CD28-B7-Immunglobulin-Superfamilie gehört [8]. Dieses CTLA-4-Protein bindet mit hoher Affinität an die kostimulatorischen B7-Moleküle auf der Antigen-präsentierenden Zelle, was eine weitergehende Aktivierung der T-Zelle unterbindet. Dieser Mechanismus hilft, eine unerwünschte, dauerhafte Immunantwort zu vermeiden.

Die Bindung von blockierenden Antikörpern an das CTLA-4-Molekül führt schließlich zu einer Aufhebung dieser Abschaltung der T-Zellen und erlaubt somit die Entwicklung von starken und lang anhaltenden Immunantworten. Da dieser Mechanismus sich nicht auf tumorspezifische T-Zellen beschränkt, kann es beim Einsatz der CTLA-4 Antikörper neben der erwünschten antitumoralen Wirkung auch zu unerwünschten Autoimmunphänomenen wie Colitis, Hepatitis und Hypophysitis kommen, welche sich aber in der Regel gut beherrschen lassen. Die bisherigen klinischen Studien wurden mit zwei verschiedenen Antikörpern durchgeführt: Ipilimumab (IgG1; MDX-010; Plasmahalbwertszeit von 12-14 Tagen) und Tremelimumab (IgG2; CP-675,206; Plasmahalbwertszeit von 22 Tagen). Nachdem in Phase-II-Studien zum Teil lang anhaltende und auch komplette Remissionen bei bis zu 15\% der Patienten beobachtet wurden, waren die Ergebnisse einer Phase-III-Studie bei 655 Patienten mit nicht resezierbarem Melanom im Stadium IIIC oder IV zum Vergleich von Tremelimumab mit Standardchemotherapie (Temozolomid oder Dacarbazin) mit Spannung erwartet worden. Dabei zeigte sich in Bezug auf das mittlere Gesamtüberleben (11,8 Monate für Tremelimumab versus 10,7 Monate für Chemotherapie) allerdings kein signifikanter Vorteil für den Anti-CTLA4-Antikörper [9]. Eine weitere Phase-III-Studie zur Wirksamkeit einer Kombination von Ipilimumab und Dacarbazin hat die Rekrutierung beendet, die Ergebnisse stehen noch aus. Schließlich wird die Wirk- 


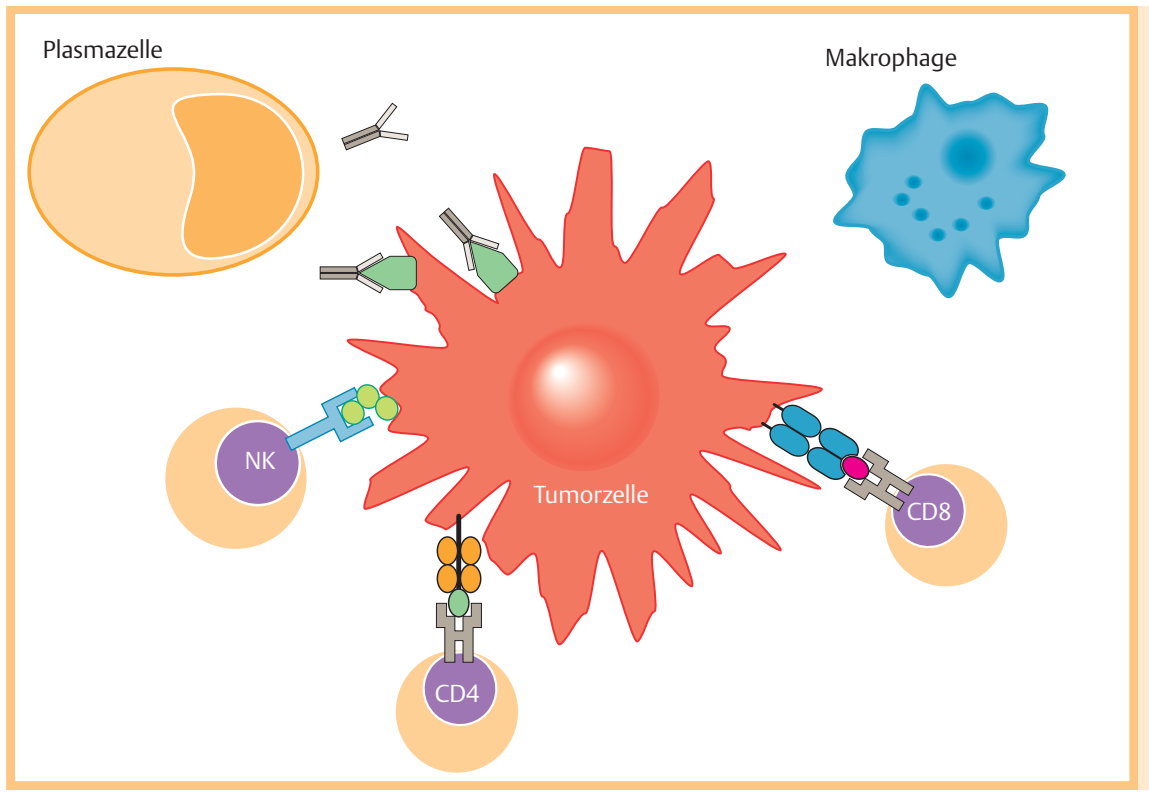

Abb. 1 In der antitumoralen Immunität spielen nicht nur tumorreaktive T-Zellen, welche HLA-Peptid-Komplexe auf der Oberfläche der Tumorzellen erkennen, eine wichtige Rolle, sondern auch weitere Zellen des Immunsystems wie NK-Zellen, Plasmazellen und Makrophagen. NK-Zellen erkennen Tumorzellen, welche die Expression von HLA-Molekülen herabreguliert haben und somit nicht mehr von T-Zellen erkannt werden können. Plasmazellen sezernieren Antikörper, welche an die Tumorzellen binden und dadurch die Antikörper-vermittelte Lyse durch NK-Zellen auslösen können. Durch die Ausschüttung von Interferon-gamma durch T-Zellen und NK-Zellen können wiederum Makrophagen aktiviert werden, um die Tumorzellen zu phagozytieren. samkeit von Ipilimumab auch in der adjuvanten Situation nach Resektion von Lymphknotenmetastasen im Rahmen einer Phase-III-Studie der EORTC-Melanomgruppe untersucht.

Das therapeutische Potenzial der Anti-CTLA4-Antikörper kann derzeit nicht abschließend beurteilt werden. Es wird darauf ankommen, aus den vorhandenen Daten Patientengruppen zu identifizieren, welche mit hoher Wahrscheinlichkeit von dieser Behandlung profitieren könnten. Möglicherweise spielen präexistente, antitumorale T-Zell-Antworten eine Rolle, möglicherweise ist auch die Kombination von Anti-CTLA4-Antikörpern mit definierten Tumorantigenen ein interessantes Konzept, um die induzierte Immunantwort zielgerichteter zu steuern.

\section{Tumorvakzination}

$\nabla$

Ziel einer jeden Tumorvakzine ist die Induktion einer gegen den Tumor gerichteten Immunantwort, welche zur Zerstörung der Tumorzellen führt. Dabei geht es insbesondere um die Aktivierung von tumorreaktiven T-Zellen, wobei neben den CD8+-zytotoxischen T-Zellen auch die CD4+-Helfer-T-Zellen eine wichtige Rolle spielen. Wahrscheinlich sind weitere Zellen des Immunsystems wie NK-Zellen, NKT-Zellen, B-Lymphozyten und Makrophagen ebenfalls wichtig für eine möglichst breite und effektive Immunantwort gegen den Tumor ( $\bullet$ Abb. 1). Die molekulare Charakterisierung von Tumorantigenen verbunden mit den Fortschritten der Biochemie und der Molekularbiologie ermöglichte den Einsatz von definierten Impfstoffen in Form von Peptiden, Proteinen oder Nukleinsäuren. Da diese allein wenig immunogen sind, werden sie mit sogenannten Adjuvanzien kombiniert, um eine potente Immunantwort zu induzieren. Als Adjuvanzien werden zumeist körperfremde Moleküle z.B. bakterieller, viraler oder pflanzlicher Natur verwendet. Als Beispiel seien die sogenannten $\mathrm{CpG}$-Oligonukleotide genannt. Es handelt sich dabei um nichtmethylierte Cytidin-Guanosin-Dinukleotide mit bestimmten flankierenden Basensequenzen, wie sie auch in mikrobieller DNA vorkommen. Diese Oligonukleotide binden an spezifische Rezeptoren auf Antigen-präsentierenden Zellen und imitieren eine bakterielle Infektion, was wiederum zur Aktivierung der Immunzellen führt.

\section{Klinische Studien}

Während eine Vielzahl von unterschiedlichen Vakzinationsstrategien in Phase-I/II-Studien untersucht worden sind bzw. weiterhin untersucht werden, wurden nur wenige Ansätze bisher im Rahmen von randomisierten Phase-III-Studien evaluiert. Diese konnten regelmäßig die zuvor beobachteten positiven Effekte nicht bestätigen.

\section{Ganglioside \\ $\nabla$}

Aufbauend auf der Beobachtung, dass Patienten mit im Serum nachgewiesenen Antikörpern gegen ein bestimmtes, auf Melanomzellen vorkommendes Sphingolipid, das sogenannte Gangliosid GM2, eine verbesserte Prognose haben, wurde Anfang der 90er-Jahre eine Vakzination mit GM2 in Kombination mit Bacillus Calmette Guerin (BCG) als Adjuvans auf ihre Wirksamkeit im Vergleich zu BCG allein bei Patienten mit R0-Resektion von regionären Lymphknotenmetastasen untersucht. Es zeigte sich kein Vorteil für den Gangliosid-Arm im Vergleich zur BCG-Impfung allein [10]. Eine weitere Phase-III-Studie zum Vergleich von Hochdosis-Interferon mit einer Gangliosidvakzine bei $880 \mathrm{~Pa}-$ tienten mit einer Tumordicke $>4 \mathrm{~mm}$ oder nach R0-Resektion von regionären Lymphknotenmetastasen wurde vorzeitig abgebrochen, da sich bei einer Zwischenanalyse nach einer medianen Nachbeobachtung von 16 Monaten ein signifikanter Überlebensvorteil für die Interferon-behandelten Patienten zeigte [11].

\section{Allogene Tumorzellen/Tumorzelllysate}

Ein anderer Ansatz, welcher in Phase-III-Studien untersucht wurde, ist die Impfung mit bestrahlten, allogenen Tumorzellen oder Tumorzelllysaten, welche den theoretischen Vorteil bieten, dass man den Patienten gegen eine Vielzahl von Antigenen immunisiert.

Für Canvaxin ${ }^{\circledR}$, eine aus 3 allogenen, bestrahlten Melanomzelllinien hergestellte Melanomvakzine, konnte in zwei Phase-IIIStudien zur adjuvanten Vakzination von Melanompatienten im 
Stadium III bzw. IV kein Überlebensvorteil im Vergleich zu Plazebo gezeigt werden, sodass die Studien vorzeitig beendet wurden. Ein weiterer Impfstoff, der aus einem Lysat aus 2 allogenen Melanomzelllinien plus einem Adjuvans besteht $\left(\right.$ Melacine $^{\circledR}$ ), wurde in der SWOG 9035-Studie bei insgesamt 689 Patienten nach Resektion eines Primärmelanoms mit einer Tumordicke zwischen 1,5 und $4,0 \mathrm{~mm}$ im Vergleich zur alleinigen Beobachtung auf seine Wirksamkeit untersucht. Auch in dieser Studie konnte kein Vorteil in Bezug auf das krankheitsfreie Überleben gezeigt werden [12]. Eine Subgruppenanalyse zeigte allerdings einen Vorteil für Patienten mit einem bestimmten HLA-Typ. So waren nach 5 Jahren $80 \%$ der Patienten mit HLA-A2 und/oder HLA-C3-Expression ohne Rezidiv im Vergleich zu $40 \%$ in der Beobachtungsgruppe.

\section{Definierte Tumorantigene \\ $\nabla$}

Das erste beim Melanom entdeckte Tumorantigen wurde 1991 charakterisiert und Melanoma Antigen 1 (MAGE-1) genannt [13]. In der Folge wurden zahlreiche weitere Mitglieder der MAGEGenfamilie identifiziert, so auch MAGE-3. MAGE-3 wird von ca. $65 \%$ der Melanome exprimiert und kommt auch in einer Vielzahl von anderen Tumoren vor, nicht aber in Normalgeweben, mit der Ausnahme von Hoden (Spermatogonien) und Plazenta (Trophoblasten). Spermatogonien und Trophoblasten exprimieren allerdings keine HLA-Moleküle und können daher nicht von T-Lymphozyten erkannt werden. Somit gilt MAGE-3 als tumorspezifisches Antigen. Immunisierungsansätze mit MAGE-3 wurden bislang gut vertragen, wenngleich bei Verwendung von potenten Adjuvanzien auch mit Autoimmunphänomenen gerechnet werden muss, welche einer unspezifischen Aktivierung des Immunsystems geschuldet sind. In der metastasierten Situation zeigten einzelne Patienten, welche mit MAGE-3-Peptiden oder auch dem gesamten Protein geimpft wurden, sowohl partielle als auch komplette Remissionen. Allerdings waren die Ansprechraten nicht zuletzt aufgrund der hohen Tumorlast und der damit verbundenen Immunsuppression gering.

Aufbauend auf diesen Studienergebnissen hat man die Erkenntnis gewonnen, dass eine Krebsimpfung besonders Erfolg versprechend bei einer geringen Tumorlast, also in einem frühen Erkrankungsstadium, ist. Eine weitere Voraussetzung für die Wirksamkeit eines solchen Ansatzes ist die Expression des Zielantigens durch den Tumor. In diesem Sinne stellt die Immuntherapie eine in hohem Maße zielgerichtete Therapie dar. Basierend auf diesen Überlegungen wurde die DERMA-Studie (Adjuvant immunotherapy with MAGE-3 in melanoma) konzipiert. In dieser plazebokontrollierten Studie wird die Wirksamkeit einer Vakzination mit einem rekombinanten MAGE-3-Protein kombiniert mit einem Adjuvans bei Patienten mit operativ entfernten, makroskopischen Lymphknotenmetastasen untersucht. Einschlussbedingung ist der Nachweis der Expression von MAGE-A3 durch die Tumorzellen, es sollen ca. 1300 Patienten randomisiert werden.

\section{Dendritische Zellen \\ $\nabla$}

Dendritische Zellen sind professionelle Antigen-präsentierende Zellen, welche unter anderem aus Monozyten des peripheren Blutes gewonnen werden und ex vivo mit Tumorantigenen beladen werden können. Sie gelten als optimales Adjuvans zur Induktion Antigen-spezifischer Immunantworten. Durch die Impfung mit Antigen-beladenen dendritischen Zellen konnten folglich nicht nur beachtliche Immunantworten, sondern auch die Rückbildung von Metastasen bei Patienten mit zum Teil weit fortgeschrittener Tumorerkrankung erzielt werden. Allerdings waren auch hier die Ergebnisse einer randomisierten Phase-III-Studie zum Vergleich der Vakzination mit Peptid-beladenen dendritischen Zellen mit der Standardchemotherapie mit Dacarbazin enttäuschend, da beide Therapiearme mit einer Ansprechrate unter $10 \%$ gleich schlecht abschnitten [14].

Dabei fiel ähnlich wie bei der Studie mit Canvaxin ${ }^{\circledR}$ auf, dass zumindest eine Subgruppe mit einem bestimmten HLA-Typ (HLAA2.1+, B44-) im Vakzinationsarm ein längeres Überleben zeigte.

\section{Fazit}

\section{$\nabla$}

Es konnte wiederholt gezeigt werden, dass durch immuntherapeutische Ansätze eine antitumorale Immunantwort induziert werden kann, welche teilweise auch mit objektiven Tumorremissionen einhergeht. In randomisierten Phase-III-Studien gelang es bisher jedoch nicht, einen signifikanten Therapieerfolg zu demonstrieren. Dafür gibt es wohl mehrere Gründe. Zum einen mangelte es bislang an geeigneten Surrogatparametern, welche das Ansprechen auf eine Vakzination vorhersagen können. Mögliche Ansätze für eine geeignete Patientenselektion werden unter anderem in der DERMA-Studie umgesetzt. Hier werden im adjuvanten Ansatz Patienten mit minimaler Tumorlast vakziniert, nachdem zuvor die Expression des Antigens MAGE-3 durch den Tumor nachgewiesen worden ist. Auf eine HLA-Typisierung der Patienten wurde verzichtet, da man für die Vakzine das gesamte Antigen verwendet und nicht nur einzelne HLA-restringierte Peptide. Trotzdem wäre es nach Kenntniss der oben erwähnten Studienergebnisse interessant gewesen, die HLA-A2-Expression als Stratifikationsfaktor zu berücksichtigen.

Zudem waren die Patientenkollektive in einigen der bisher durchgeführten Studien nicht groß genug, um einen kleinen Therapienutzen zu zeigen. Auch dies wurde in der DERMA-Studie berücksichtigt. Hier sollen 1300 Patienten inkludiert werden, beinahe doppelt so viele wie in der oben zitierten Melacine ${ }^{\circledR}$-Studie.

Interessenkonflikt: Der Autor gibt an, dass kein Interessenkonflikt besteht.

\section{Abstract}

\section{Immunotherapy for Melanoma}

\section{$\nabla$}

Melanoma represents an immunogenic tumour who can be recognized and destroyed by the immune system. This antitumour immunity is not only mediated by T cells but also by B cells and NK cells. Due to continuous research on the molecular mechanisms underlying the interactions between tumour cells and immune cells new immunotherapeutic approaches are further optimized. However, a significant clinical benefit for patients still needs to be achieved. Better selection of patients, who might respond to immunotherapy by determing the HLA status and the antigen expression profile of the tumor might help to overcome the therapeutic resistance of melanoma. 


\section{Literatur}

1 Grob JJ, Dreno B, de La SP et al. Randomised trial of interferon alpha-2a as adjuvant therapy in resected primary melanoma thicker than $1.5 \mathrm{~mm}$ without clinically detectable node metastases. French Cooperative Group on Melanoma. Lancet 1998; 351: 1905-1910

2 Pehamberger $H$, Soyer HP, Steiner A et al. Adjuvant interferon alfa-2a treatment in resected primary stage II cutaneous melanoma. Austrian Malignant Melanoma Cooperative Group. J Clin Oncol 1998; 16: 1425 1429

3 Eggermont AM, Suciu S, Santinami M et al. Adjuvant therapy with pegylated interferon alfa-2b versus observation alone in resected stage III melanoma: final results of EORTC 18991, a randomised phase III trial. Lancet 2008; 372: 117 - 126

4 Kirkwood JM, Strawderman MH, Ernstoff MS et al. Interferon alfa-2b adjuvant therapy of high-risk resected cutaneous melanoma: the Eastern Cooperative Oncology Group Trial EST 1684. J Clin Oncol 1996; 14: $7-17$

5 Kirkwood JM, Manola J, Ibrahim J, Sondak V, Ernstoff MS, Rao U. A pooled analysis of eastern cooperative oncology group and intergroup trials of adjuvant high-dose interferon for melanoma. Clin Cancer Res 2004; 10: 1670 - 1677

6 Wheatley $K$, Ives $N$, Hancock B et al. Does adjuvant interferon-alpha for high-risk melanoma provide a worthwhile benefit? A meta-analysis of the randomised trials. Cancer Treat Rev 2003; 29: 241 - 252

7 Ascierto PA, Kirkwood JM. Adjuvant therapy of melanoma with interferon: lessons of the past decade. J Transl Med 2008; 6: 62
8 Greenwald RJ, Freeman GJ, Sharpe AH. The B7 family revisited. Annu Rev Immunol 2005; 23: 515- 548

9 Ribas A, Hauschild A, Kefford R et al. Phase III, open-label, randomized, comparative study of tremelimumab (CP-675,206) and chemotherapy (temozolomide [TMZ] or dacarbazine [DTIC]) in patients with advanced melanoma. J Clin Oncol 2008; 26: LBA9011

10 Livingston PO, Wong GY, Adluri S et al. Improved survival in stage III melanoma patients with GM2 antibodies: a randomized trial of adjuvant vaccination with GM2 ganglioside. J Clin Oncol 1994; 12: 1036 1044

11 Kirkwood JM, Ibrahim JG, Sosman JA et al. High-dose interferon alfa-2b significantly prolongs relapse-free and overall survival compared with the GM2-KLH/QS-21 vaccine in patients with resected stage IIB-III melanoma: results of intergroup trial E1694/S9512/C509801. J Clin Oncol 2001; 19: 2370-2380

12 Sondak VK, Liu PY, Tuthill RJ et al. Adjuvant immunotherapy of resected, intermediate-thickness, node-negative melanoma with an allogeneic tumor vaccine: overall results of a randomized trial of the Southwest Oncology Group. J Clin Oncol 2002; 20: 2058-2066

13 van der Bruggen $P$, Traversari C, Chomez $P$ et al. A gene encoding an antigen recognized by cytolytic $\mathrm{T}$ lymphocytes on a human melanoma. Science 1991; 254: $1643-1647$

14 Schadendorf D, Ugurel S, Schuler-Thurner B et al. Dacarbazine (DTIC) versus vaccination with autologous peptide-pulsed dendritic cells (DC) in first-line treatment of patients with metastatic melanoma: a randomized phase III trial of the DC study group of the DeCOG. Ann Oncol 2006; 17: $563-570$ 